J Neurol Neurosurg Psychiatry 2004;75(Suppl II):ii43-ii50. doi: 10.1136/jnnp.2004.040378

$\mathrm{N}$ eurological manifestations of cancer are common, disabling, and often multifactorial (table 1). The concept that malignant disease can cause damage to the nervous system above and beyond that caused by direct or metastatic infiltration is familiar to all clinicians looking after cancer patients. These "remote effects" or paraneoplastic manifestations of cancer include metabolic and endocrine syndromes such as hypercalcaemia, and the syndrome of inappropriate ADH (antidiuretic hormone) secretion. Paraneoplastic neurological disorders (PNDs) are remote effects of systemic malignancies that affect the nervous system. The term PND is reserved for those disorders that are caused by an autoimmune response directed against antigens common to the tumour and nerve cells.

PNDs are much less common than direct, metastatic, and treatment related complications of cancer, but are nevertheless important because they cause severe neurological morbidity and mortality and frequently present to the neurologist in a patient without a known malignancy. Because of the relative rarity of PND, neurological dysfunction should only be regarded as paraneoplastic if a particular neoplasm associates with a remote but specific effect on the nervous system more frequently than would be expected by chance. For example, subacute cerebellar ataxia in the setting of ovarian cancer is sufficiently characteristic to be called paraneoplastic cerebellar degeneration, as long as other causes have been ruled out. However, carpal tunnel syndrome in a patient with cancer is not paraneoplastic because both are reasonably common conditions which may co-exist in the same patient.

\title{
INCIDENCE OF PND
}

There have only been a few studies addressing the incidence and prevalence of PND in the overall cancer population. A number of studies have focused on either certain cancers typically associated with PND—for example, small cell lung cancer (SCLC) ${ }^{1}$ - or on specific anti-neuronal antibodies and their neurological and tumour associations.

The incidence of PND depends on the stringency of the criteria used for the diagnosis. In the first systematic study of PND, Croft and Wilkinson coined the term "carcinomatous neuromyopathy" to describe patients with cancer who had neuromuscular abnormalities, particularly proximal muscle weakness and peripheral neuropathies. They found that $4 \%$ of women with breast cancer, $16 \%$ of men with lung cancer, and $6.6 \%$ of patients with all cancers had evidence of a PND compared with 1-2\% of age matched controls. ${ }^{2}$ Among 1465 patients with all different cancers, only 15 had cerebellar degeneration and eight had sensory neuronopathy. These results imply that the incidence of specific and disabling neurological disorders is much lower. The most frequent PND is Lambert-Eaton myasthenic syndrome (LEMS) which occurs in $2-3 \%$ of patients with SCLC. ${ }^{1}$ In patients with gynaecological cancer PNDs are even less common, occurring in about 1 per 1000 new cases. Overall PNDs affect fewer than $1 \%$ of patients with cancer and so these conditions pale into insignificance when compared with the frequency of metastatic and iatrogenic complications of cancer. Therefore, a PND should be diagnosed in a patient with cancer only after investigations have ruled out other possible causes.

\section{Age and sex ratio}

Patients with PND usually present in later life. In a recent surveillance study of unselected cases from the UK, the median age of onset was 66 years (range 30-77 years) and only seven patients (11\%) were less than 50 years at presentation. ${ }^{3}$ Similarly in a study of 200 patients from France and Spain with anti-Hu associated paraneoplastic encephalomyelitis (PEM), the median age at presentation was 63 years. ${ }^{4}$ In patients with paraneoplastic cerebellar degeneration (PCD) the median age of patients is 64, 67, and 65 years, depending on the associated paraneoplastic neuronal autoantibody (PNA). Patients with Hodgkin's disease and anti-Tr or anti-mGluRl antibodies are slightly younger. ${ }^{5}$ These data taken together suggest that PNDs rarely occur in young people-if they do, the tumours to think about include Hodgkin's disease (PCD), testicular 
Table 1 Neurological manifestations of malignant disease

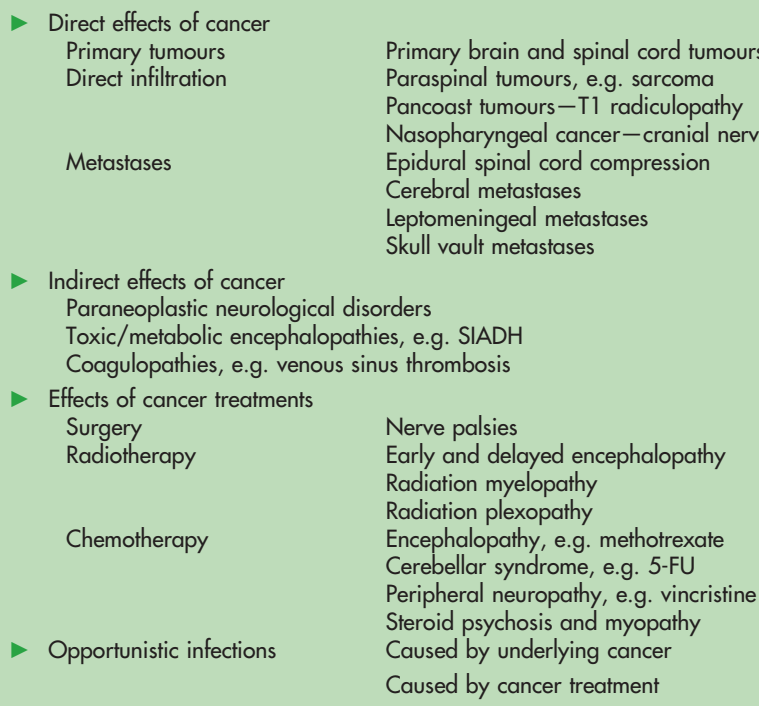

5-FU, 5-fluorouracil; PML, progressive multifocal leucoencephalopathy; SIADH, syndrome of inappropriate ADH secretion.

cancer (brainstem and limbic encephalitis), breast cancer (many different syndromes) and, in children, neuroblastoma (opsoclonus-myoclonus).

The sex ratio of patients with PND is conflicting. In the UK study, there was a striking female preponderance (3.2:1) that could not be accounted for by the association with breast and gynaecological tumours alone since the corrected ratio was still 2.3:1. In contrast, Graus' study cited above found a male predominance (3:1) perhaps reflecting the lower incidence of SCLC in European women when compared with women in the USA. ${ }^{4}$ What consistently emerges from a number of studies, however, is that women are more likely than men to develop PEM if they have SCLC.

\section{Tumour associations}

Almost any malignant tumour except (interestingly) brain tumours can give rise to almost any PND. The most common associated cancers are SCLC, breast, gynaecological cancers and lymphoma. I have come across a number of patients with malignant neuroendocrine tumours presenting with a PND perhaps reflecting their embryological origins from the neural crest. As SCLC only occurs in smokers, it is important to consider the diagnosis of PND in any patient with a history of smoking and unexplained neurological dysfunction. Tumours of mesenchymal origin-for example, sarcomas-are rarely associated with PND.

It is important to stress that in about $20 \%$ of cases, no cancer is ever found, even at postmortem examination, probably reflecting the successful control of tumour growth and metastasis by the host immune system in patients with PND. There have also been anecdotal reports of spontaneous resolution of lung cancer in patients with PND, but this is rare.

\section{WHEN TO SUSPECT PND}

PND can affect almost any part of the nervous system. The syndrome can be remarkably focal-for example, paraneoplastic cerebellar degeneration (PCD)—or multifocal such as limbic and brainstem encephalitis with sensory neuronopathy (PEM/PSN). The onset of central nervous system
(CNS) disorders is usually rapid and may in some cases be so acute as to be mistaken for a stroke. I have recently seen a patient who developed a cerebellar syndrome over 2-3 days and six months later was diagnosed with a malignant neuroendocrine tumour of the stomach. More commonly, the natural history is one of subacute progression over weeks to months. PND may render a previously fit patient bedbound within a few weeks and in the context of a cerebellar syndrome gives rise to a very short differential diagnosis consisting of drug toxicity (usually evident from the history), prion disease (usually associated with cognitive decline), or paraneoplasia. In contrast LEMS may be more insidious.

Table 2 lists the CNS manifestations of PND by anatomical site. This attempt at classification is somewhat artificial as PNDs are commonly multifocal. Indeed, it is this multifocality which often suggests the diagnosis of PND, as few other disorders in neurology have such a predilection for specific neurological locations such as the dorsal root ganglia, the cerebellum, brainstem, and limbic system. Other multifocal disorders to consider in the differential diagnosis include vasculitides, inflammatory and granulomatous CNS disorders, and meningeal infiltrations.

Table 2 Paraneoplastic neurological disease affecting the central nervous system

Brain and retina

Paraneoplastic encephalomyelitis \pm sensory neuronopathy (PEM/ PSN)

Limbic encephalitis (LE)

Brainstem encephalitis (BE)

Paraneoplastic encephalomyelitis with rigidity

Paraneoplastic cerebellar degeneration (PCD)

Paraneoplastic opsoclonus-myoclonus (POM) Paraneoplastic retinal degeneration

- Spinal cord, dorsal root ganglia, and anterior horn cells Myelitis

Acute necrotising myelopathy

Dorsal root ganglionitis (paraneoplastic sensory neuronopathy) Stiff person syndrome (SPS)

Motor neurone syndromes 


\section{PND affecting the CNS}

Paraneoplastic encephalomyelitis (PEM)

This is one of the most common PNDs affecting the CNS and is characterised by a multifocal inflammatory process with a predilection for limbic and brainstem structures. This may occur with or without sensory neuronopathy, autonomic neuropathy, and neuromuscular disease. The various components of PEM are now discussed in isolation although, as indicated above, they may occur as part of a more complex neurological presentation.

\section{Limbic encephalitis}

Limbic encephalitis (LE) is usually seen in association with SCLC and presents with amnesia, seizures, agitation, and an altered mental state, often on the background of anxiety and depression. The main finding on examination is of severe impairment of recent memory progressing to dementia which on occasion may improve dramatically with treatment of the underlying tumour. Investigations are usually non-specificthere may be a cerebrospinal fluid (CSF) pleocytosis and an elevated protein concentration. Magnetic resonance imaging (MRI) is usually normal although occasionally there is high signal change within one or both medial temporal lobes (fig 1). Pathological changes affecting limbic and basal ganglia structures include neuronal cell loss, reactive microglial proliferation, and perivascular lymphocytic infiltration. A variety of paraneoplastic neuronal autoantibodies (PNAs) are associated with LE, including anti-Hu, anti-Mal and Ma2, and anti CV2. Patients with anti-Ma antibodies may have associated hypothalamic involvement and germ cell tumours of the testis. The main differential diagnosis is of herpes simplex encephalitis, although this is usually a more acute illness. Rarely patients who have had bone marrow transplantation may develop a limbic encephalitis caused by reactivation of human herpesvirus-6-an important diagnosis not to miss as this can be treated with foscarnet.

\section{Brainstem encephalitis}

Brainstem encephalitis (BE) is similar to LE in that it is often associated with clinical and pathological evidence of

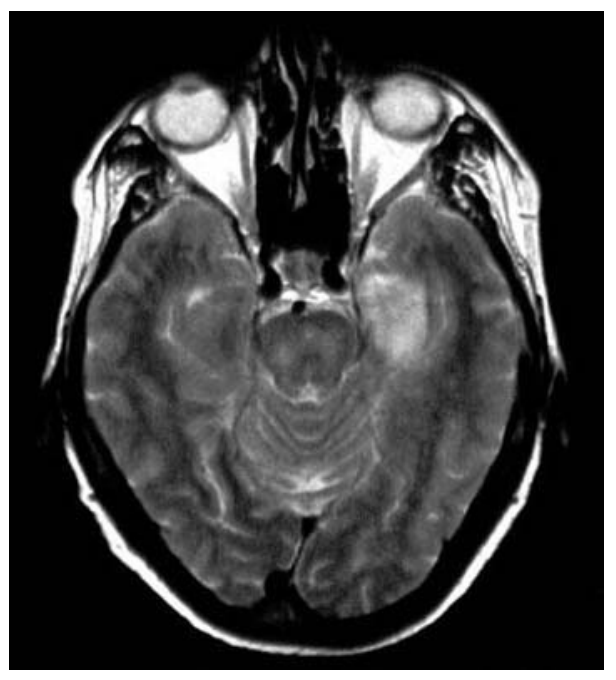

Figure 1 Axial T2 weighted magnetic resonance image (MRI) scan of a patient with ant-Hu associated limbic encephalitis showing high signal in the left medial temporal lobe. encephalomyelitis elsewhere within the nervous system, but it can occur in isolation or as the predominant clinical manifestation of PND. The clinical syndrome is heterogeneous and is characterised by a combination of cranial nerve palsies, long tract signs, and cerebellar ataxia. Less common features include movement disorders, such as parkinsonism, chorea, jaw opening dystonia, and myoclonus. Occasionally it can present with neurogenic hypoventilation causing recurrent episodes of central respiratory failure. As with LE, investigations are rarely helpful-high signal change may be seen within the brainstem and basal ganglia and the CSF may be inflammatory with oligoclonal bands. These disorders are usually life threatening and rarely respond to treatment.

Paraneoplastic encephalomyelitis with rigidity This is a rare syndrome characterised by truncal and limb rigidity, stimulus sensitive myoclonus, and inflammatory pathology in the brainstem and spinal cord. Not all cases are paraneoplastic, but a strong pointer to a paraneoplastic aetiology is the presence of anti-amphiphysin antibodies in the patient's serum.

\section{Paraneoplastic cerebellar degeneration}

Paraneoplastic cerebellar degeneration (PCD) is seen in a variety of tumours, most commonly breast and gynaecological cancer, lung cancer, and Hodgkin's disease. Typically the disorder starts with an unsteady gait which progresses rapidly to a pancerebellar syndrome rendering the patient bed bound, severely dysarthric, and tremulous within a few weeks of onset. Complex eye movement abnormalities are usually seen and nystagmus is frequently downbeating. Most patients complain of vertigo, diplopia, dysphagia, and occasionally oscillopsia. The condition is usually symmetrical but I have seen two cases with striking asymmetry. It is usually suspected when imaging of the posterior fossa is unremarkable in the presence of severe cerebellar ataxia. Eventually MRI shows cerebellar atrophy. Other investigations-for example, CSF examination-are rarely helpful but are useful in ruling out leptomeningeal metastases which can occasionally present with isolated gait ataxia. These patients usually stabilise after a few months and rarely respond to treatment, even when the underlying tumour is detected and removed. However, spontaneous resolution has been reported particularly when associated with Hodgkin's disease. Pathologically there is severe and sometimes complete loss of Purkinje cells with relatively minor thinning of the molecular layer. The deep cerebellar nuclei are spared except in cases of encephalomyelitis in which case there are more extensive inflammatory changes seen. PCD is associated with a number of anti-neuronal antibodies including anti-Yo (breast and gynaecological), anti-Hu (SCLC), anti-Tr, and anti-mGluR (Hodgkin's disease).

\section{Paraneoplastic opsoclonus-myoclonus}

Paraneoplastic opsoclonus-myoclonus (POM) is an extremely rare syndrome seen in young children with neuroblastoma (dancing eyes syndrome) and adults with breast cancer and SCLC. It is characterised by opsoclonus, a disorder of saccadic eye movements causing continuous involuntary arrhythmic multidirectional high amplitude saccades. This may occur in isolation but more commonly is associated with action myoclonus affecting the whole body, including the diaphragm and palate. Some patients also have cerebellar 
ataxia. This syndrome is associated with cancer in about $20 \%$ of adults-it can also be seen in viral infections, drug overdose, and as part of a postinfectious encephalitis. As with other PND affecting the CNS, the standard investigations are usually normal or non-specifically normal. Several autoantibodies have been described in POM, particularly anti-Ri antibodies (breast, SCLC) and anti-neurofilament antibodies in children with neuroblastoma.

\section{Paraneoplastic retinal degeneration}

PND can manifest as visual failure through a variety of mechanisms. Retinal photoreceptors, rods, cones, and optic nerves can all be affected. Paraneoplastic retinal degeneration, otherwise known as cancer associated retinopathy, is usually seen in SCLC and gynaecological tumours, and is associated with antibodies against recoverin, a photoreceptor protein. Visual loss is painless and bilateral and may present initially with loss of colour vision, night blindness, and photosensitivity. Visual testing reveals loss of visual acuity, and field testing shows peripheral and ring scotomas. There may be arteriolar narrowing and mottling of the fundus caused by changes in the retinal pigment epithelium. The electroretinogram is abnormal.

\section{Myelitis and acute necrotising myelopathy}

Myelopathy, occurring as the main neurological syndrome, is less common. As with LE and BE, it may be part of a more widespread encephalomyelitis. Rarely, it can progress with astonishing rapidity in the context of a necrotising myelopathy. Pathologically there is extensive necrosis of both white and grey matter, most notably in the thoracic segments.

\section{Dorsal root ganglionitis (paraneoplastic sensory neuronopathy)}

Paraneoplastic sensory neuronopathy (PSN) is characterised by subacute, rapidly progressive, asymmetric and often painful, sensory symptoms dominated by profound proprioceptive loss affecting upper limbs more than lower limbs. All sensory modalities are affected-in contrast motor function is preserved. Nerve conduction studies show low sensory nerve action potentials but normal motor studies. The CSF is typically inflammatory, particularly if the neuronopathy is associated with encephalomyelitis. The differential diagnosis includes Sjögren's syndrome and cisplatin toxicity. The pathology is centred around the dorsal root ganglia with loss of ganglion cells and lymphocytic infiltrates (fig 2).

\section{Stiff person syndrome}

Stiff person syndrome (SPS) is characterised clinically by axial and proximal lower limb stiffness and spasms, and electrophysiologically by continuous motor unit activity. This disorder is more commonly non-paraneoplastic and is associated with anti-GAD antibodies, but in a minority of cases it may be the presenting feature of breast cancer with anti-amphiphysin antibodies. Paraneoplastic encephalomyelitis with rigidity (see above) is the most aggressive variant of SPS.

\section{Motor neurone syndromes}

There is considerable controversy as to whether motor neurone disease (MND) can be a paraneoplastic syndrome-this has been clarified by Forsyth and colleagues ${ }^{6}$ who identified three groups of patients: the first with rapidly progressive MND and anti-Hu antibodies; the second, women

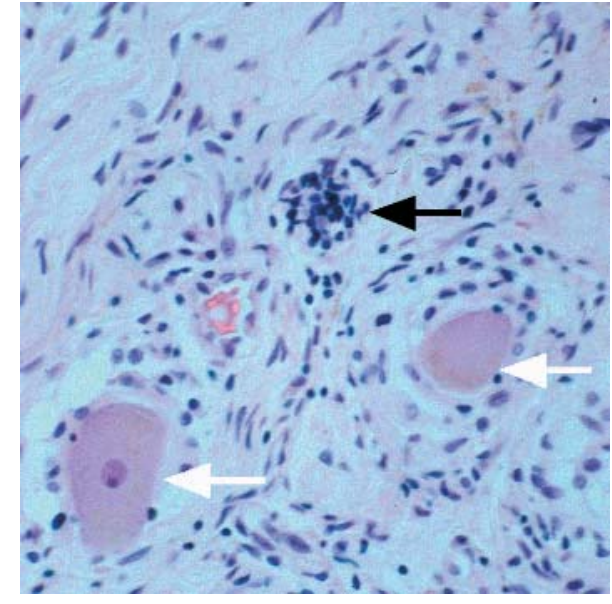

Figure 2 Sensory neuronopathy caused by dorsal root ganglionitis showing a hypercellular cluster of lymphocytes (nodule of Nageotte) indicating active neuronophagia and ganglion cell degeneration (black arrow). White arrows indicate surviving ganglion cells.

with primary lateral sclerosis and breast cancer; and the third with MND occurring some years after a diagnosis of cancer. It seems likely that the first two groups are paraneoplastic- the third probably not, merely reflecting the occurrence of two reasonably common diseases of older age in the same patient separated in time.

\section{PND affecting the peripheral nervous system}

The peripheral nervous system is more commonly involved than the CNS and the range of disorders is summarised in table 3. Mild distal sensorimotor neuropathies are quite common in patients with cancer and are not necessarily paraneoplastic - other factors to consider include metabolic, nutritional, and treatment related toxicity. In patients presenting with a severe peripheral, predominantly sensory neuropathy, in whom standard investigations are negative, about $10 \%$ will eventually be diagnosed with cancer, particularly if there is rapid progression to severe disability and the absence of regeneration in a sural nerve biopsy.

\section{Sensory and sensorimotor neuropathy}

Paraneoplastic sensory and sensorimotor neuropathies are well recognised in many different cancers. Nerve conduction studies show a mixed sensory and motor axonal neuropathy-occasional patients may have typical demyelinating

Table 3 Paraneoplastic neurological disease affecting the peripheral nervous system

Peripheral nerve

Sensory neuropathy (axonal or demyelinating)

Sensorimotor neuropathy (axonal or demyelinating)

Acute inflammatory demyelinating neuropathy (AIDP)

Motor neuronopathy

Microvasculitic neuropathy

Autonomic neuropathy

Neuromyotonia

- Neuromuscular junction

Lambert-Eaton myasthenic syndrome (LEMS)

Myasthenia gravis

- Muscle

Polymyositis/dermatomyositis (PM/DM)

Acute necrotising myopathy 
features and respond to intravenous immune globulin. Patients with chronic inflammatory demyelinating polyradiculopathy (CIDP) with atypical features including resistance to first line treatments, an unusually aggressive course, or the presence of myopathy and long tract signs should be investigated for an underlying cancer. The CSF is usually acellular in contrast with sensory neuronopathy, but the protein is usually raised. An unusual "osteosclerotic" form of myeloma may cause a combination of endocrine, dermatological, and neurological features encapsulated within the acronym POEMS (polyneuropathy, organomegaly, endocrinopathy, $M$ protein, skin changes). The neuropathy is classically demyelinating.

\section{Acute inflammatory demyelinating polyradiculoneuropathy}

Motor neuropathies are much less common than sensory neuronopathies in PND. Patients with Hodgkin's disease can present with acute inflammatory demyelinating polyradiculoneuropathy (AIDP), a syndrome clinically and electrophysiologically indistinguishable from Guillain-Barré syndrome. Similarly brachial plexitis (neuralgic amyotrophy) has also been occasionally reported in association with lymphoma, but the evidence for a true paraneoplastic association is poor.

\section{Motor neuronopathy}

A subacute lower motor neuronopathy is occasionally seen with non-Hodgkin's lymphoma presenting as a slowly progressive lower motor neurone syndrome affecting the lower limbs.

\section{Microvasculitic neuropathy}

Vasculitis is occasionally associated with haematological malignancies and usually presents with cutaneous signs. Paraneoplastic vasculitic neuropathy is very unusual and occurs most commonly in the setting of B cell lymphomas.

\section{Autonomic neuropathy}

Patients with underlying SCLC and anti-Hu antibodies may present with a progressive autonomic neuropathy affecting the bowel leading to intestinal pseudo-obstruction and gastric paresis. This is usually part of an encephalomyelitis.

\section{Neuromyotonia}

Acquired neuromyotonia is an unusual manifestation of cancer characterised by muscle cramps, stiffness and twitching, sweating, and abnormal relaxation after voluntary contraction. It is distinguishable from normal muscle cramps by the finding of prolonged bursts of high frequency repetitive muscle action potentials on the electromyogram (EMG). Antibodies against voltage gated potassium channels are detected in patients' sera and are associated with thymomas and SCLC. The disorder may also be autoimmune.

\section{Lambert-Eaton myasthenic syndrome}

Lambert-Eaton myasthenic syndrome (LEMS) is caused by SCLC in $60 \%$ of cases and is characterised by fatiguable proximal weakness, predominantly affecting lower limbs, in association with autonomic disturbance such as impotence, constipation, and dry mouth. The paraneoplastic syndrome is usually more severe than the autoimmune form but is electrophysiologically indistinguishable. The characteristic finding, consistent with presynaptic neuromuscular dysfunction, is of small compound muscle action potentials which decrement at low frequency stimulation but increment at high frequency stimulation. The diagnosis of LEMS is confirmed by finding antibodies against $\mathrm{P} / \mathrm{Q}$ type voltage gated calcium channels (VGCC). Some patients with LEMS also have a cerebellar syndrome, and some patients with cerebellar syndromes but without LEMS have anti-VGCC antibodies.

\section{Myasthenia gravis}

Myasthenia gravis is the prototypical autoimmune disease affecting the postsynaptic acetylcholine receptors at the neuromuscular junction. In about $10 \%$ of cases there is an underlying thymoma and therefore myasthenia gravis can be considered to be a paraneoplastic disease. No other tumours are associated with myasthenia gravis.

\section{Polymyositis/dermatomyositis}

Myositis is a common paraneoplastic manifestation either in the form of polymyositis or dermatomyositis. In general, patients with paraneoplastic myositis are older and have a more severe course compared to their autoimmune counterparts. Dermatomyositis presenting in the elderly is more likely to be paraneoplastic than polymyositis.

\section{Acute necrotising myopathy}

Acute necrotising myopathy is a rare myopathy with necrosis of muscle fibres. It is proximal, rapidly progressive, associated with rhabdomyolysis, and usually fatal.

\section{HOW TO CONFIRM PND}

Standard investigations including blood tests, MRI scanning, CSF analysis, and clinical neurophysiology testing are rarely helpful in confirming that a patient has a PND. They should be regarded as investigations which help to rule out other differential diagnoses such as structural lesions, meningeal infiltration, other autoimmune disease, particularly primary Sjögren's syndrome, and inflammatory, vasculitic or granulomatous CNS diseases.

Once a PND is suspected and the above tests are negative, diagnosis rests either on the demonstration of an underlying malignancy or the presence of circulating PNAs. Either may be positive when the other is negative. If both are negative, this does still does not rule out the diagnosis of PND and repeat investigations are warranted every six months or so until an alternative diagnosis emerges.

\section{Paraneoplastic neuronal autoantibodies}

In 1965 Croft and co-workers reported the discovery of organ specific antibodies reacting against whole brain in four out of five patients with bronchial carcinoma and sensory neuronopathy. Since then, the concept of an autoimmune hypothesis to explain the pathogenesis of PND has been supported by the identification of numerous PNAs in the serum and CSF of patients with PND. To date, no other group of conditions has been identified where PNAs are consistently found and so their detection remains the cornerstone of diagnosis. These antibodies react with different intracellular and extracellular antigens ranging from nuclear proteins (for example, $\mathrm{Hu}$ antigens) to membrane channels (for example, VGCC). They are thought to arise as a result of aberrant expression of antigens, common to tumours and neurones. This leads to loss of tolerance and the induction of an immune response that reacts with both tumour and normal neuronal tissue. Whether or not these antibodies are pathogenic is a moot 
point; in diseases affecting the neuromuscular junction-for example, LEMS and myasthenia gravis-there is no doubt that these antibodies are directly responsible for the clinical syndrome. In contrast, however, it has not been possible to reproduce PND affecting the CNS either with active immunisation or passive transfer, even intrathecally, of relevant neuronal antigens or antibodies. There is increasing evidence that cytotoxic $\mathrm{T}$ cell mechanisms play a pathogenic role, although it has not yet been possible to reproduce an animal model of these diseases.

Table 4 lists the more common PNAs and describes their molecular autoantigens and associated clinical syndromes and tumours. This is an ever expanding list-it is not necessary to memorise these antibodies, as a competent laboratory will be able to detect almost all of them by immunohistochemical staining of patients' sera against mammalian nervous system followed by Western blotting against homogenates of different parts of the nervous system in order to characterise the antibody.

Detection of certain antibodies identifies patients with specific clinical syndromes and tumours-for example, antiTr antibodies in Hodgkin's disease and cerebellar degeneration. However, certain antibodies-for example, anti-Hu antibodies-are found in a variety of clinical phenotypes and associated tumours (fig 3). Furthermore, many patients with definite PND do not have anti-neuronal antibodies and so a negative screen does not exclude the diagnosis.

\section{Identification of underlying tumour}

Once a PND is suspected, the search is on for an underlying tumour. This may be difficult as the tumour may be smaller than the limits of resolution of conventional computed tomographic (CT) scanning. Many patients are thus subjected to a number of invasive and fruitless investigations including CT thorax, abdomen, pelvis, bronchoscopy, and colonoscopy without any tumour being found. I therefore advocate the early use of whole body ${ }^{18 \mathrm{~F}}$ fluoro-2-deoxyglucose-positron emission tomography (FDG-PET) scanning in the investigation of patients with suspected PND as it may pick up small abnormalities and guide further imaging to specific regions of the body (fig 4). We have a number of patients who had normal CT and ultrasound imaging but an abnormal PET scan which subsequently led to the correct diagnosis. $^{7}$

\section{HOW TO MANAGE PND}

In general, PNDs of the CNS are resistant to immunomodulatory treatments except in a few isolated cases. Often the patient is so severely disabled by the time the diagnosis is made that no specific treatment is effective. The rapid progression early on followed by stabilisation suggests that by the time a PND is diagnosed, irreversible neurological damage has already occurred. A few case reports suggest benefit from intravenous immune globulin when treatment is started within a few weeks of onset. Similarly trials of other immunomodulatory treatments such as corticosteroids, azathioprine, cyclophosphamide, and plasma exchange have generally been unsuccessful.

For this reason, discovery and treatment of the underlying tumour remains the mainstay of management. In a study of 200 patients with anti-Hu associated paraneoplastic encephalomyelitis, treatment of the tumour, with or without immunosuppression, was an independent predictor of improvement/stabilisation of the disease. ${ }^{4}$ We have also found that in an unselected series of 63 patients who were followed up for at least one year, only treatment of the tumour was associated with an improved chance of neurological stabilisation or improvement. ${ }^{3}$ Spontaneous improvements and even regression of an underlying cancer have been reported, but this is rare.

In contrast, both paraneoplastic and non-paraneoplastic LEMS respond well to immunosuppressive treatment with steroids, intravenous immune globulin, and plasma exchange. $^{8}$ However, because of the theoretical risk of hastening tumour growth and dissemination in the paraneoplastic forms of the disease, it is essential to treat an underlying tumour first as this will often improve the neurological symptoms concurrently. In patients in whom no tumour is found, the treatment is the same as for nonparaneoplastic LEMS.

Table 4 Paraneoplastic autoantibodies (PNAs), relevant autoantigens, characteristic staining patterns, and associated paraneoplastic neuronal disorders (PNDs) and tumours

\begin{tabular}{|c|c|c|c|c|}
\hline PNA & Neuronal auto antigen(s) & Immunocytochemical staining pattern & PND & Tumour \\
\hline Anti-Hu (ANNA-1) & $\begin{array}{l}35-40 \mathrm{kDa} \text { neuron specific RNA } \\
\text { binding proteins }\end{array}$ & $\begin{array}{l}\text { All neuronal nuclei including myenteric plexus, } \\
\text { weaker cytoplasmic staining }\end{array}$ & PEM, PCD, PSN & SCLC, other carcinomas \\
\hline Anti-Ri (ANNA-2) & $\begin{array}{l}55 \text { and } 80 \mathrm{kDa} \text { RNA neuronal } \\
\text { binding protein }\end{array}$ & $\begin{array}{l}\text { All CNS neuronal nuclei with nucleolar sparing, } \\
\text { not DRG neurones (in contrast to } \mathrm{Hu} \text { ) }\end{array}$ & POM, PCD, BE, & Breast, SCLC, gynaecological \\
\hline ANNA-3 & $170 \mathrm{kDa}$ & $\begin{array}{l}\text { Purkinje-cell cytoplasm and nucleus; renal } \\
\text { glomerular podocytes }\end{array}$ & LE, PCD, PSN & SCLC, oesophageal \\
\hline Anti-Yo (PCA-1) & $\begin{array}{l}34 \text { and } 62 \mathrm{kDa} \text { DNA binding, } \\
\text { gene transcription regulators }\end{array}$ & Purkinje cell cytoplasm and axons & $\begin{array}{l}P C D \text {, peripheral } \\
\text { neuropathy (rare) }\end{array}$ & $\begin{array}{l}\text { Breast and gynaecological, } \\
\text { oesophageal }\end{array}$ \\
\hline Anti-PCA 2 & $280 \mathrm{kDa}$ & Purkinje-cell cytoplasm and other neurones & PEM, PCD, LEMS & SCLC \\
\hline Anti-Ma 1 & $40 \mathrm{kDa}$ & Nucleus & $\mathrm{BE}, \mathrm{PCD}$ & Lung cancer \\
\hline Anti-Ma 2 (Ta) & $41.5 \mathrm{kDa}$ & Nucleus, perikaryon & $\mathrm{LE}, \mathrm{BE}$ & Testicular cancer \\
\hline Anti-Tr & Unknown antigen & $\begin{array}{l}\text { Purkinje cell cytoplasm, fine granular staining } \\
\text { in molecular layer; all nuclei of CNS }\end{array}$ & PCD & Hodgkin's disease \\
\hline Anti-mGluR1 & Metabotropic glutamate receptor & Purkinje cell cytoplasm, climbing fibres & PCD & Hodgkin's disease \\
\hline Anti-amphiphysin & $128 \mathrm{kDa}$ synaptic vesicle protein & Presynaptic nerve terminals of CNS & SPS, PEM & Breast, SCLC \\
\hline Anti-VGKC & Voltage gated potassium channel & Peripheral nerve & Neuromyotonia & Thymoma, SCLC \\
\hline Anti-VGCC & $\begin{array}{l}64 \mathrm{kDa} P / \mathrm{Q} \text { voltage gated calcium } \\
\text { channel }\end{array}$ & Pre-synaptic neuromuscular junction & LEMS, PCD & SCLC \\
\hline Anti-ACh R & ACh receptor & Post-synaptic neuromuscular junction & MG & Thymoma \\
\hline
\end{tabular}

ACh, acetylcholine; BE, brainstem encephalitis; CNS, central nervous system; LE, limbic encephalitis; LEMS, Lambert-Eaton myasthenic syndrome; MG, myasthenia gravis; PCD, paraneoplastic cerebellar degeneration; PEM, paraneoplastic encephalomyelitis; PNA, paraneoplastic neuronal autoantibody; PND, paraneoplastic neurological disorder; POM, paraneoplastic opsoclonus-myoclonus; PSN, paraneoplastic sensory neuronopathy; SCLC, small cell lung carcinoma; SPS, stiff person syndrome. 

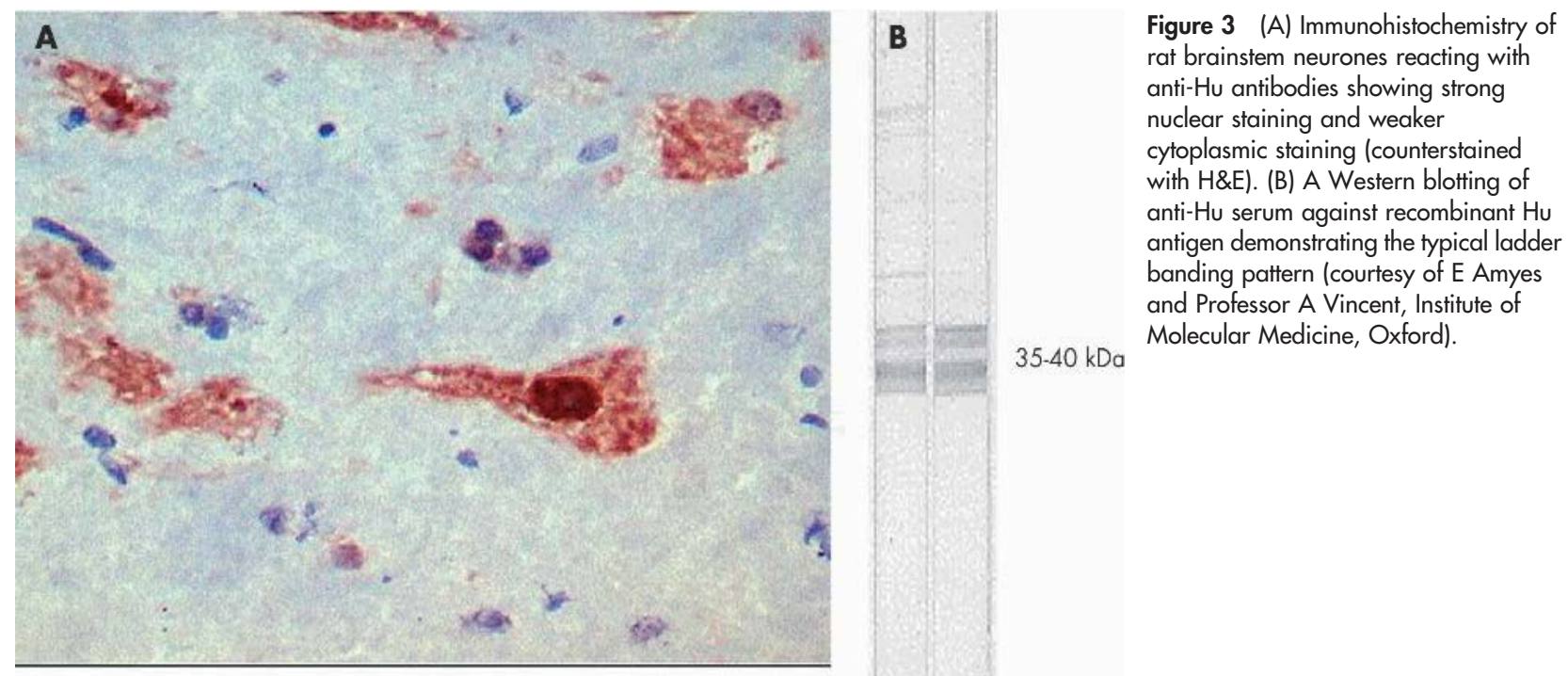

\section{PROGNOSIS}

There is a clear distinction in prognosis between neuromuscular junction disorders, particularly LEMS, which carry a good prognosis and the CNS disorders which are usually inexorably progressive. There are a few case reports of

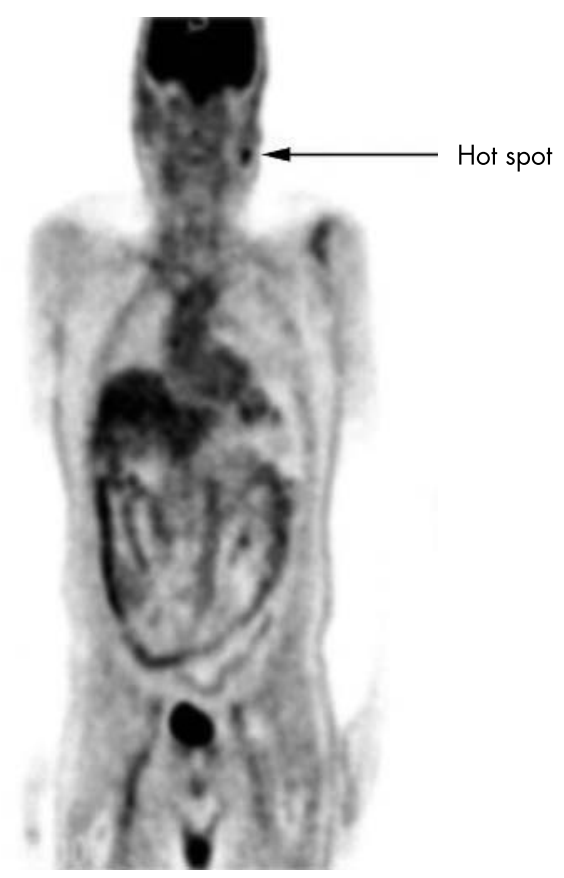

Figure 4 Whole body FDG-PET scan in a 69 year old man with cerebellar degeneration of unknown cause. Extensive investigations had failed to reveal the site of a tumour. The scan reveals a single hot spot under the left jaw which was biopsied and shown to be Hodgkin's disease. dramatic improvements once a tumour has been identified and removed-usually in patients with LE-however, the majority of patients stabilise after a few months and remain severely disabled for the rest of their life even when the tumour is "cured". Presumably this reflects underlying neuronal cell death and so if treatment is to have any benefit it needs to be given expeditiously before a critical mass of neurones have been destroyed.

In contrast patients with tumours associated with PND frequently fare better than patients with similar tumours without PND. This may be due to lead time bias in diagnosing the tumour at an early presymptomatic stage, but there is some evidence that neurologically normal patients with SCLC who had low titres of anti-Hu antibodies have a much better survival than age and stage matched patients without these antibodies. $^{9}$

It seems reasonable therefore to investigate whether the anti-tumour response in patients with PND can be used to treat tumours without damaging the nervous system.

\section{CONCLUSIONS}

PNDs are rare but serious remote manifestations of a variety of tumours. They are clinically heterogeneous and are usually diagnosed on the basis of detection of circulating PNA and/or an underlying tumour. Management is still unsatisfactory, particularly of the CNS disorders.

\section{REFERENCES}

1 Elrington GM, Murray NM, Spiro SG, et al. Neurological paraneoplastic syndromes in patients with small cell lung cancer. A prospective survey of 150 patients. J Neurol Neurosurg Psychiatry 1991:54:764-7.

2 Croft $P$, Wilkinson M. The incidence of carcinomatous neuromyopathy in patients with various types of carcinomas. Brain 1965;88:427-34.

3 Candler PM, Hart PE, Barnett $M$, et al. A follow-up study of patients with paraneoplastic neurological disease in the United Kingdom. J Neurol Neurosurg Psychiatry (in press). 
4 Graus F, Keime-Guibert F, Rene R, et al. Anti-Hu-associated paraneoplastic encephalomyelitis: analysis of 200 patients. Brain $2001 ; 124: 1138-48$.

5 Shams'ili S, Grefkens J, de Leeuw B, et al. Paraneoplastic cerebellar degeneration associated with anti-neuronal antibodies: analysis of 50 patients. Brain 2003;126:1409-18.

6 Forsyth PA, Dalmau J, Graus F, et al. Motor neuron syndromes in cancer patients. Ann Neurol 1997;41:703-5.

\section{ii50} scanning in the diagnosis of paraneoplastic neurological disorders. Brain $2001 ; 124: 2223-31$.
8 Newsom-Davis J. Therapy in myasthenia gravis and Lambert-Eaton myasthenic syndrome. Semin Neurol 2003;23:191-8.

9 Graus F, Dalmau J, Rene R, et al. Anti-Hu antibodies in patients with small-cell lung cancer: association with complete response to therapy and improved survival. J Clin Oncol 1997;15:2866-72.

\section{KEY REFERENCE}

1 Darnell RB, Posner JB. Paraneoplastic syndromes involving the nervous system. N Engl J Med 2003;349:1543-54. 